\title{
ARTICLE
}

\section{Epilepsy and neuropsychiatric comorbidities}

\author{
Niruj Agrawal \& Suren Govender
}

\begin{abstract}
Niruj Agrawal is a consultant neuropsychiatrist at St George's Hospital in London and an honorary senior lecturer with St George's, University of London. He is responsible for providing tertiary care neuropsychiatry service to the population of south-west London and Surrey. He has a special interest in neuropsychiatric disorders associated with epilepsy and other neurological disorders. Suren Govender is a consultant in old age psychiatry at Surrey an Borders Partnership NHS Foundation Trust. He has a special interest in neuropsychiatry and mental health informatics.

Correspondence Dr Niruj Agrawal, Clare House, St George's Hospital, London SW17 00T, UK. Email: Niruj. Agrawal@swlstg-tr.nhs.uk
\end{abstract}

\begin{abstract}
SUMMARY
Epilepsy is a common neurological condition with wide-ranging neuropsychiatric manifestations. The relationship between epilepsy and psychiatry has been recognised for centuries. However, the wide range of neuropsychiatric comorbidities and their extent is only now beginning to be appreciated. The impact of these comorbidities on patients' help-seeking behaviour, seizure control and quality of life suggests that early detection and treatment are of paramount importance. Clinical issues in relation to accurate recognition and appropriate management of neuropsychiatric conditions in epilepsy are discussed.
\end{abstract}

\section{DECLARATION OF INTEREST}

None.

Descriptions of epileptic fits can be found in ancient texts spanning two millennia in a number of civilisations, including Egypt, China, India and Babylonia. Hippocrates (born $460 \mathrm{Bc}$ ) first hypothesised that epilepsy was due to brain disease and not supernatural causes as was

BOX 1 Definitions and terminology

Epilepsy

A tendency to unprovoked and recurrent seizures.

Aura

Simple partial seizures, which may or may not progress into another seizure type. They are often mistakenly considered as a prodrome for a seizure.

\section{Seizure/ictus}

An abnormal paroxysmal electrical discharge of cerebral neurons.

Fit

The physical manifestation of a seizure.

Generalised seizures

Epileptic seizures that manifest immediately and spread bilaterally through the cerebral cortex. They may present with or without tonic-clonic convulsions, and primary generalised seizures are usually not associated with auras.

Partial or focal seizures

Epileptic seizures that start in a specific area of the brain and then spread. A partial seizure may become secondarily generalised if it spreads to both hemispheres.

\section{Prodrome}

A preictal phenomenon characterised by subjective or objective awareness of a clinical change that heralds the onset of a seizure, but that does not form part of it.

A transient period of central nervous system abnormality, which becomes apparent after the ictal phase.

\section{Postictal phase}

previously believed. However, this was soon forgotten and it was not until the late 19th century that a clearer understanding of epilepsy began to emerge, following experimentation in evoking epileptic seizures. Years later, further work by Gibbs, Lennox, Penfield and Jasper consolidated understanding of epilepsy as a medical illness (Goldensohn 1997).

Epilepsy is a neurological condition characterised by recurrent seizures. It affects $0.4-1 \%$ of the general population, with a higher incidence in males than females (World Health Organization 2005). The terminology and definitions used in the field of epilepsy are summarised in Box 1. Box 2 shows the most commonly used international classification system, developed by the International League Against Epilepsy (1989).

The diagnosis of epilepsy remains clinical, with particular emphasis on careful history-taking. There is no diagnostic test or investigation for epilepsy. The electroencephalogram (EEG) is an extremely useful investigation but must be used in conjunction with clinical assessment. In addition, home videos and video-EEG telemetry and brain imaging (e.g. computed tomography or magnetic resonance imaging) can be used to clarify the diagnosis and the underlying cause. Diagnosis should be made by a specialist (National Collaborating Centre for Mental Health 2004).

Anti-epileptic drugs are the mainstay of treatment. There are now over 15 drugs licensed in the UK for treating and preventing seizures, and about $50 \%$ of patients will be seizure-free after treatment with the first anti-epileptic drugs prescribed. This falls to about $20 \%$ after a second medication, leaving approximately 30\% treatment refractory (Kwan 2000).

\section{Neuropsychiatric manifestations and comorbidities}

The association between epilepsy and mental health problems is reported in ancient texts. Hercules is said to have suffered from epilepsy and 'madness'. For centuries, the care provisions for patients with both mental health problems and epilepsy reflected this overlap. However, despite long-standing recognition of this association and 


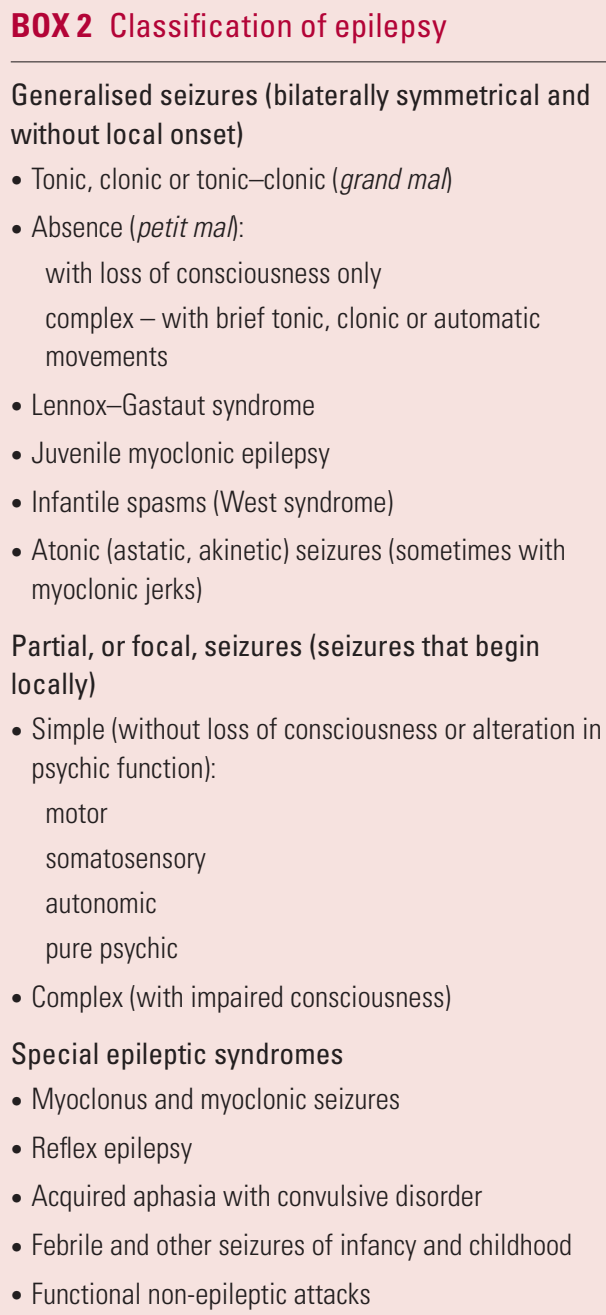

(Adapted from Ropper 2005)

advancement in the care of patients with epilepsy, neuropsychiatric disorders in epilepsy are still often missed.

Patients with intellectual disability, particularly severe intellectual disability, may have different neuropsychiatric presentations from those without intellectual disability. This review focuses primarily on epilepsy in adults without intellectual disability.

There is a dearth of systematic epidemiological data regarding the prevalence of psychiatric disorder in epilepsy. Rates of neuropsychiatric manifestations depend on the clinical settings, with the highest rates seen in specialist epilepsy clinics. In addition, screening method and diagnostic issues affect the reported rates. Available data suggest that psychiatric comorbidity occurs in $20-40 \%$ of patients with epilepsy, with an even higher incidence in people with treatment-resistant epilepsy and temporal lobe epilepsy. An additional $5-20 \%$ of patients attending epilepsy clinics show

evidence of functional non-epileptic attacks. A range of psychiatric problems can occur in people with epilepsy, including affective disorders, psychotic illnesses and personality change. However, most studies have focused on the link between epilepsy and depression and consequently there is a lack of epidemiological data on the association between epilepsy and psychiatric disorders as a whole (Devinsky 2003). The figures quoted are therefore likely to be an underestimate. Box 3 describes risk factors for psychiatric comorbidity in epilepsy.

Early recognition and treatment of neuropsychiatric comorbidities in epilepsy is important. Psychiatric conditions in epilepsy are associated with greater usage of healthcare services (Zaber 2007) and significantly impaired self-reported health status. Furthermore, remarkably consistent data have emerged on the association of decreased quality of life in epilepsy (measured by the Quality of Life in Epilepsy-89 inventory) with depression (measured by the Beck Depression Inventory) (Viikinsalo 2000; Boylan 2004). Interestingly, no such association is found with seizure frequency, unless there is complete remission, or with any other clinical or demographic variable. Similar data in relation to anxiety disorders in epilepsy are emerging. This suggests that identifying and treating psychiatric comorbidities in epilepsy could be one of the ways to improve a patient's health status and quality of life, and may help reduce healthcare costs.

A complex mathematical model used to analyse the relationship between epilepsy and depression shows that, although high seizure frequency could be associated with depression, depression predicts higher seizure frequency independently (Thaper 2005), indicating a reciprocal relationship. This suggests that treating affective symptoms associated with epilepsy could improve seizure control.

BOX 3 Risk factors for psychopathology in epilepsy

\section{Clinical factors}

Age at onset of epilepsy, duration of disorder, type and frequency of seizure, the site of the brain affected, and interictal and ictal EEG abnormalities.

\section{Psychosocial factors}

Chronicity of disease, low socioeconomic status, low educational level, social stigma, difficulties adjusting to the consequences of illness, external locus of control, fear of seizures, overprotection by families, legal limitations (e.g. not being permitted to hold a driving licence), low self-esteem.

\section{Biological factors}

Neuropathological damage to areas of the brain associated with psychic functioning (e.g. the amygdala, limbic system, frontal cortex, basal ganglia), emotional and cognitive side-effects induced by some anti-epileptic drugs and medication-induced low serum folate level, which may indirectly affect mental state.

(Adapted from Torta 1999) 
Diagnosing psychiatric comorbidities can be difficult in patients with epilepsy. The effect of anti-epileptic medications and symptoms of epilepsy itself can either mask or mimic features of psychiatric problems. Patients with epilepsy may present with psychiatric symptoms which may not neatly fit in with commonly used diagnostic categories. Treating these conditions may also pose complexities and dilemmas. Hence, a good awareness of all of these issues will go a long way in improving the quality of care of patients with epilepsy and mental health problems.

\section{Depression}

Depression is the most common psychiatric disorder in patients with epilepsy and a significant cause of morbidity. Reported rates of depression in epilepsy are $20-55 \%$ for patients with recurrent seizures, depending on the study (Kanner 2003). Precise data on incidence and prevalence are lacking; this could be due to differing methodologies and sample populations in studies, underreporting of depressive symptomatology by patients and underdiagnosis by clinicians. There is growing evidence of a biological link between depression and epilepsy, and depleted biogenic amines and gamma aminobutyric acid may be significant factors in the development of both disorders (Barry 2003). Depression seems to be more common in epilepsy than in other chronic medical conditions such as asthma, indicating it is not just an emotional reaction to a disabling chronic condition (Ettinger 2005).

Kanner et al (2000) reported that $60 \%$ of patients with depression and epilepsy had shown symptoms of depression for over a year before any treatment was recommended. The delay in recognising the need for treatment was not related to severity of depression.

Features of depression such as sleep disturbance, appetite change, lack of concentration and reduced energy levels are less useful clinically in diagnosing depression, owing to the intrinsic effect of epilepsy and the side-effects of anti-epileptic medications. More emphasis should be given to lack of interest, anhedonia and depressive cognitions in addition to subjective sustained sadness.

\section{Preictal depression}

This is considered to manifest as prodromal moods of depression or irritability that occur hours to days before a seizure and may last up to a few days. Symptoms typically resolve post-seizure. It is unclear whether the depressive symptoms are part of seizure manifestation or whether causative factors for depressive symptoms reduce the seizure threshold (Lambert 1999).

\section{Ictal depression}

Dysthymia or depressive symptoms (commonly, feelings of anhedonia, guilt and suicidal thoughts) may be manifestations of seizure activity. They are typically sudden in onset, brief, stereotypical and associated with other ictal phenomena (such as automatisms, fear and hallucinations).

\section{Postictal depression}

Kanner (2003) studied over 100 consecutive patients with poorly controlled partial seizure disorders and found that $43 \%$ had a median of five postictal symptoms of depression. The median duration for $66 \%$ of the symptoms was $24 \mathrm{~h}$. Interestingly, postictal depression may also be associated with postictal psychoses.

\section{Interictal depression}

Prueter \& Norra (2005) reported interictal depression as the most common presentation of depression in epilepsy. Although the exact prevalence is unknown, they estimated it to be between $20 \%$ and $70 \%$, depending on the study.

Diagnosis can be made using either the ICD-10 (World Health Organization 1992) or DSM-IV (American Psychiatric Association 1994) criteria for depression, and patients often present with a clinical picture of major depression or dysthymia. Interictal depression often follows a chronic course, interspersed with short, symptom-free phases. Other notable symptoms include atypical pain, phases of euphoric/dysphoric affect, anxiety and phobic symptoms.

Blumer et al (2004) described interictal dysphoric disorder of epilepsy, which presents with recurrent brief episodes of mild depressive symptoms. This disorder was initially thought to be specific to patients with epilepsy but recent studies show the presence of similar episodes in other neurological conditions (Mula 2008).

\section{Treatment}

The treatment of depressive illness in epilepsy remains poorly explored despite the high prevalence. It is surprising that the most recent double-blind placebo-controlled study was over 20 years ago (Robertson 1985). It involved 42 patients with depression and epilepsy who were entered into an antidepressant trial of amitriptyline, nomifensine and placebo. Current treatment recommendations are largely based on anecdotal evidence and clinical experience.

\section{Preictal, ictal and postictal depression}

These depressive conditions are usually selflimiting and do not require treatment. Improved 
seizure control may reduce the frequency of symptoms. However, if the preictal or postictal depression is prolonged and distressing, it may be affecting the epileptic threshold. In such circumstances, treatment of depression should be considered. Blumer (1992) proposed low-dose antidepressant medication with optimal control of the epilepsy as the treatment of choice for postictal depression.

\section{Interictal and comorbid depression}

The use of antidepressant medication causes much anxiety for practitioners treating patients with epilepsy and comorbid depressive disorders. Prueter \& Norra's (2005) thorough review suggests the following guidance for the treatment of depression. It is thought that rapid dose escalation and higher overall doses are more likely to induce increased seizure activity, and the review recommends citalopram and sertraline as first-line agents because of the minimal pharmacokinetic interaction with anti-epileptic drugs. Generally, selective serotonin reuptake inhibitors (SSRIs), venlafaxine and nefazodone appear less likely to induce seizures, and mirtazapine may be suitable for agitated patients who require a more sedating treatment. Monoamine oxidase inhibitors seem to have a lower propensity to affect epileptic threshold, whereas tricyclic antidepressants seem to have a higher one. It is suggested that antidepressant doses should be increased slowly and at small increments. Although antidepressant medications lower the seizure threshold, this effect may be balanced out, as untreated depression may also lower the epileptic threshold. Hence, antidepressants still have a clear role in the treatment of depression in epilepsy.

Consideration should be given to changing anti-epileptic medication with known depressive effects to that with mood-stabilising properties, for example carbamazepine, valproate or lamotrigine. It is also worth noting that the anti-epileptics topiramate, zonisamide, phenytoin, phenobarbitone and ethosuximide should be avoided in depression as they may exacerbate depressive symptoms.

Pharmacokinetic interactions must also be taken into account in treating this group of patients. Enzyme inducers such as carbamazepine, phenytoin, phenobarbital and primidone may cause increased metabolism of antidepressant drugs, whereas some SSRIs (fluoxetine, fluvoxamine and paroxetine) inhibit the cytochrome P450 (CYP450) system and may increase the serum levels of some anti-epileptic drugs. These factors are important and need to be considered when prescribing antidepressant drugs. Electroconvulsive therapy (ECT) is not contraindicated in patients with epilepsy and can be considered in individuals with severe, treatment-resistant depression. ${ }^{\dagger}$

Finally, psychological treatment should be offered to newly diagnosed patients and efforts should be made to educate them and their families about epilepsy, to correct false beliefs about the condition and to improve awareness of potential psychiatric comorbidities. Cognitive-behavioural therapy (CBT) may help the patient to come to terms with the disorder and develop healthy coping strategies. There is currently limited data supporting the use of psychotherapy. Frequent seizures, reduced concentration, fatigue and cognitive impairment may limit the role of psychological treatment in some patients.

\section{Suicide}

Studies have shown a two- to threefold increase in the standardised mortality ratio in patients with epilepsy (Ficker 2000). In a study by Hauser et al (1980), the cause of death in an epilepsy cohort by accidents and suicide was $6 \%$.

A strong association has been reported between the risk of suicide and onset of epilepsy at an early age, particularly during adolescence (Nilsson 2002). Overall, the risk of suicide is 2.4 times higher in patients with epilepsy, 11-12 times higher in those with epilepsy and anxiety or psychosis, and 32 times higher in those with epilepsy and depression (Christensen 2007).

\section{Anxiety}

Very little attention has been given to distinguishing between the features of anxiety occurring in epilepsy or the subtypes of anxiety disorders in epilepsy populations. Consequently, the incidence and prevalence of these disorders are unclear. One large cross-sectional population-based study using primary care records showed an $11 \%$ rate of anxiety disorders in 5834 people who had epilepsy compared with $5.6 \%$ in 831163 people without epilepsy (Gaitatzis 2004).

\section{Postictal anxiety}

Postictal anxiety typically occurs shortly after a seizure and can be associated with postictal dysphoria or depression. Occasionally, anxiety is the only postictal symptom experienced; however, a combination of mood and anxiety symptoms is more common. This is usually related to seizure activity and is most likely to be self-limiting. No specific treatment other than reassurance is generally required.

\author{
${ }^{\dagger}$ Allan Scott discusses ECT for \\ depression is in this issue: see pp. \\ 15-22. Ed.
}




\section{Interictal anxiety}

Interictal anxiety is common and the fears (phobic anxiety) often relate to the perceived risk of personal injury and brain damage, and having seizures in unfamiliar situations. The phenomenology for interictal anxiety is not clearly defined, and this is possibly related to the difficulty in making a distinction between independent comorbid anxiety (i.e. anxiety is not a manifestation of underlying undetected seizure semiology) and interictal anxiety relating to underlying undetected seizure semiology.

\section{Other risk factors}

A community study has shown that female gender, younger chronological age, divorce or separation, low educational attainment and unemployment are associated with increased anxiety in patients with epilepsy (Mensah 2007).

\section{Treatment}

A systematic review of anxiety in patients with epilepsy (Bayenburg 2005) recommended that treatment should be similar to the treatment of anxiety disorders in the general population and should focus on the following key areas: psychoeducation, relaxation, CBT and anxiolytic medication. The authors found no controlled studies of medical treatment in anxiety disorders in epilepsy. They suggest that SSRIs are the drug of choice. Reboxetine is also reported to be well tolerated, but seizures have been reported with amoxapine, maprotiline and most tricyclics.

The majority of psychotropic drugs are metabolised by four isoenzymes: CYP1A2, 3A4, 2C and 2D6 (Mula 2003). Almost all antidepressant drugs interact with different CYP450 enzymes, which can result in interactions with enzymeinducing or -inhibiting anti-epileptic drugs. Some of the newer anti-epileptic drugs (e.g. gabapentin, levetiracetam, pregabalin) have a lower risk of these types of interactions, which possibly makes them useful for co-administration with antidepressant drugs. Pregabalin is now licensed for treating generalised anxiety disorder in the UK.

Benzodiazepines should generally not be used as anxiolytics in people with epilepsy because of the danger of dependence and withdrawal seizures. However, there is one notable exception: clobazam can be used as prophylaxis for anxiety-related seizures.

\section{Psychosis}

Psychosis of epilepsy includes a range of psychotic disorders with variable phenomenology related to the seizure disorder. Psychosis of epilepsy was differentiated from schizophrenia in the 1950s, and it described paranoid delusions with visual and auditory hallucinations occurring in patients with epilepsy. Patients with psychosis of epilepsy rarely exhibit negative symptoms of schizophrenia, have a generally preserved personality and affect, and better premorbid functioning.

Psychotic experiences in the general epilepsy population are present in 0.6-7\% (Krohn 1961), and these figures can increase to 19-27\% (Devinsky 1993) in epilepsy centres. In a prospective study of psychosis and epilepsy, children with temporal lobe epilepsy had a 10\% chance of developing interictal psychoses during a 30-year follow-up, whereas the risk in the general population was about $0.8 \%$ (Torta 1999).

\section{Ictal psychosis of epilepsy}

In ictal psychosis of epilepsy, psychotic symptoms are a manifestation of an underlying epileptic seizure. Commonly encountered symptoms are hallucinations (olfactory, gustatory, visual or auditory) or delusions (particularly grandiose or paranoid). The symptoms are usually self-limiting; however, rarely (in conjunction with partial status epilepticus) they may be mistaken for schizophrenia or mania. The presence of confusion, lack of systematised delusions and prominent nonauditory hallucinations can point to the presence of ictal psychosis of epilepsy rather than other schizophrenia-like psychotic disorders.

Ictal psychosis occurs with associated epileptic brain discharges and EEG changes will often be seen. The exception is patients with simple partial status, where an EEG may be normal. In the majority of cases, the focus is in the limbic and isocortical components of the temporal lobe. In about a third of patients, the focus is extratemporal, usually occurring in the frontal or cingulate cortex (Sachdev 1998).

\section{Postictal psychosis of epilepsy}

Postictal psychosis accounts for about $25 \%$ of epileptic psychoses. A common presentation is that of a patient with partial or generalised epilepsy who experiences a cluster of complex partial or tonic-clonic seizures. Within $72 \mathrm{~h}$ of postictal confusion, affective and psychotic symptoms develop. There is often a short period of clear consciousness before symptom onset. Affective symptoms with grandiose and religious delusions and simple auditory hallucinations are common.

The duration of postictal psychosis is generally short and up to 2 weeks is considered to be the maximum. The following neurological factors are associated with increased risk of developing 
postictal psychosis: bilateral seizure foci; processes associated with bilateral limbic lesions (e.g. encephalitis, head injury); and a relative increase in seizure frequency preceding the psychotic symptoms (Devinsky 2003).

\section{Interictal psychosis of epilepsy}

The prevalence of interictal psychosis of epilepsy in populations with epilepsy is between 3 and $7 \%$ (Toone 2000). It is characterised by the presence of psychotic symptoms not temporally related to seizure activity and a mental state characterised by delusions and hallucinations in clear consciousness. Bizarre or disorganised behaviour, thought disorder, personality change, negative symptoms of schizophrenia or affective changes are not commonly seen. Before a diagnosis of interictal psychosis can be made, the following conditions need to be ruled out: anti-epileptic drug toxicity, non-convulsive status epilepticus, recent head trauma, and alcohol or drug intoxication or withdrawal.

\section{Alternative psychosis of epilepsy or forced normalisation}

This disorder, described by Landolt (1953) as forced normalisation and by Tellenbach (1965) as alternative psychosis, is characterised by an inverse relationship between seizure control and the occurrence of psychotic symptoms. The concept of forced normalisation also requires an EEG becoming more normal or entirely normal over time. It was thought to occur after years of treatment. It is more frequently recognised by European clinicians, and most commonly presents with paranoid psychosis without clouding of consciousness and with associated affective symptoms. Several anti-epileptic drugs are associated with this presentation (e.g. ethosuximide, vigabatrin, topiramate, zonisamide) and suppression of seizures is considered a possible aetiological factor. It is now recognised that although this phenomenon may be seen in a small subgroup of patients, this is not the main explanation for comorbid psychotic illness in epilepsy (Krishnamoorthy 2002).

\section{Treatment}

Ictal psychotic phenomena should be treated by optimisation of anti-epileptic medications with the aim of better seizure control. They do not warrant antipsychotic medications. A prolonged postictal or interictal psychosis would commonly require treatment with antipsychotic medication. There are no large randomised controlled trials of antipsychotic medication for psychosis of epilepsy.
In a review by Alper et al (2002), some of the general principles of treatment are discussed. The authors indicate that it is worthwhile assessing the family psychiatric history because there is some evidence to suggest that a family history of responsiveness to a specific antipsychotic is predictive of a positive response.

Antipsychotic drugs can reduce the seizure threshold to varying degrees, and the risk appears to be dose-related. Among the typical antipsychotics, those with low potency, such as the phenothiazines, are more likely to decrease the seizure threshold. Among the atypicals, risperidone and olanzapine have greater propensity to affect the seizure threshold than quetiapine, amisulpride and aripiprazole. Alper et al suggest that antipsychotics should be used at the lowest doses for the shortest time and that sudden and large dose escalations should be avoided.

Clozapine is one of the antipsychotics with a higher potential to provoke seizures. Its use in epilepsy is generally restricted to refractory psychosis in epilepsy, and the risk appears to be related to very rapid titration and is dose-dependent. At doses up to $300 \mathrm{mg} /$ day, the risk of seizures is comparable with that of other antipsychotics. Chlorpromazine and loxapine are also associated with a greater potential for lowering the seizure threshold.

Anti-epileptic drugs can be associated with onset of psychotic symptoms or depression. Psychosis has been reported with drugs such as topiramate, vigabatrin, zonisamide, tiagabine and ethosuximide. Anti-epileptic drugs can lower serum folate levels, which may manifest with depressive symptoms. Depression has occurred in association with anti-epileptic drugs such as barbiturates, ethosuximide, piracetam, tiagabine, topiramate and vigabatrin.

Potential drug interaction should also be borne in mind when prescribing anti-epileptic and psychotropic drugs together. Carbamazepine is a potent enzyme inducer and can lower plasma levels of some drugs. Selective serotonin reuptake inhibitors such as fluoxetine and paroxetine have been reported to inhibit hepatic enzymes and could result in higher plasma levels of anti-epileptic drugs. Sertraline and citalopram are relatively safe in this regard.

\section{Personality changes associated with epilepsy}

\section{Comorbid personality disorders and epilepsy}

The extent of personality disorders in association with epilepsy is $0.7-2.0 \%$ of general practice populations (Edeh 1987; Forsgren 1992). In those with partial epilepsy (particularly temporal lobe 
epilepsy), rates of personality disorder range from 13 to 35\%. A study reviewing personality disorders among individuals with treatmentrefractory epilepsy found dependent and avoidant personality disorders to be the most common (Lopez-Rodriguez 1999).

\section{Organic personality disorder}

Some people with epilepsy (particularly those with temporal lobe epilepsy) develop behavioural changes. The most common of these are:

- viscosity - a tendency for prolonged interpersonal contact, with pedantry and lack of socially appropriate ending of conversations

- hyposexuality - decreased libido and impotence (about $50 \%$ of male patients with temporal lobe epilepsy)

- religiosity - very strong preoccupation with religion and philosophy

- hypergraphia - compulsive writing

- aggression - increased incidence of interictal violence and hostility.

The combination of these traits is sometimes referred to as Gastaut-Geschwind syndrome.

An inevitable decline in personality associated with epilepsy, often referred to as epileptic personality, has been rejected. It is recognised that past reports of such a decline may have been describing the effects of psychosocial factors such as institutionalisation and biological factors such as sedative medication or brain damage.

\section{Functional non-epileptic attacks}

These are a diverse group of disorders in which paroxysmal events (fits or 'funny turns') may be mistaken for epilepsy, but are not caused by epilepsy (no seizures or epileptiform activity in the brain). They are either physiological (about 10-20\%) or psychogenic (about 80-90\%) in origin (Box 4). Diagnosis can be particularly difficult and requires specialist assessment.

\section{BOX 4 Causes of functional non-epileptic attacks}

Psychogenic causes
- Depersonalisation disorder
- Hypochondriasis
- Somatisation disorder
- Dissociative disorders
- Conversion disorders
- Panic disorders
- Factitious disorders

Physiological causes

- Syncope

- Transient ischaemic attacks

- Paroxysmal movement disorders

- Narcolepsy

- Non-epileptic myoclonus

(Adapted from Gates 2002)
A variety of terms are used to describe nonepileptic seizures: hystero-epilepsy, hysterical seizures, pseudoseizures, pseudo-epileptic seizures, non-epileptic pseudoseizures, hysterical epilepsy and non-epileptic attack disorder (Gates 2002). Many of these terms are disparaging and also are not representative of underlying pathophysiology. We recommend using the term 'functional nonepileptic attacks'. However, as the terms nonepileptic seizures or psychogenic non-epileptic seizures are widely used in the literature, we have also used these terms here.

Szaflarski et al (2000) estimated the annual incidence of psychogenic non-epileptic seizures in the general population to be about 3 per 100000 , with an estimated prevalence of 33 per 100000 (Benbadis 2000). Approximately 5-20\% of patients in epilepsy clinics have been considered to have only non-epileptic fits. About $80 \%$ of patients with psychogenic non-epileptic seizures are women and are aged between 15 and 35 years, although elderly and paediatric patients may also develop them (Shen 1990). It is important to note that non-epileptic seizures may occur in people with epilepsy (about 33\%).

The underlying mental processes leading to nonepileptic fits and their exact pathogenesis remain unclear, but a number of psychological factors/ disorders are often aetiologically relevant. These include affective/anxiety disorders, somatoform and/or dissociative disorders, abnormalities of personality (borderline personality disorder being the most common), and a history of sexual or physical abuse.

Non-epileptic fits can present in various ways. Box 5 describes some of the more common presentations, which may occur in isolation or in combination.

BOX 5 Common clinical presentation of nonepileptic fits

- Panic attack - may resemble an epileptic seizure and loss of consciousness may occur

- Avoidance attack - typically this occurs when the individual is unable to cope with a stressful situation; the person may fall to the floor and remain inert, with reduced muscle tone

- Abreactive attack - occurs as a delayed response to highly stressful experiences, and often happens towards the end of the day; hyperventilation may precede increased body tone and thrashing of the limbs

- Simulated attack - conscious or unconscious simulation of an epileptic seizure, usually involving some kind of gain 


\section{Diagnosis}

Diagnosis of functional non-epileptic seizures is essentially clinical. A detailed history is very important and it is often helpful to gain information about the initial fit, stressful life events surrounding the fit (if present), subsequent investigation, diagnosis and progression. There is no single diagnostic factor in the history or examination, but a number of factors may point towards a nonepileptic cause or psychogenic nature of the fits. For example: seizures in the presence of doctors, status epilepticus, multiple unexplained physical symptoms, multiple operations/invasive tests, history of psychiatric treatment, and a history of sexual and physical abuse.

Direct observation of fits or a video recording of the fits can be invaluable in reaching a correct diagnosis. Box 6 shows some of the observational features suggestive of non-epileptic fits, and Box 7 shows some of the common investigations. Frontal lobe seizures can commonly present with normal investigations, including normal EEG and video-EEG telemetry. Their manifestations can be behavioural and bizarre and they may be misdiagnosed as non-epileptic fits. Therefore, signs such as stereotypical nature of seizures and long clinical experience in the field is helpful in clarifying the diagnosis.

Doctors should be aware of a number of clinical factors that are considered to be of diagnostic value but which may not help in differentiating epileptic from non-epileptic fits, and may occur in both kinds of fit: tongue biting, injury, frothing, apparent progression from aura to fit to a postictal stage can all be seen in non-epileptic fits. No single clinical or historical feature is diagnostic. However, as the number of unusual features for epilepsy increases, non-epileptic fits should be considered.

\section{Treatment}

A systematic review by Gaynor et al (2009) summarised the available evidence for treatment

BOX 6 Observational features suggestive of non-epileptic fits

- Undulating motor activity

- Convulsion lasting >2 minutes

- Resisted lid opening

- Rapid postictal re-orientation

- Asynchronous limb movements

- Lack of cyanosis

- Retained awareness of surroundings in apparent generalised fits
BOX 7 Common investigations in functional non-epileptic attacks

- EEG: single interictal EEG may not be helpful in diagnosis

- Provocation procedures: common procedures used are hyperventilation, photic stimulation or sleep deprivation

- Serum prolactin: may not be very helpful and is not commonly used now because of concerns about its sensitivity and specificity

- Home videos can be a very helpful diagnostic aid

- The gold standard for diagnosis remains video-EEG telemetry; however, deep-seated focus may sometimes be missed

of functional non-epileptic attacks. Essentially, tactful presentation of diagnosis by the neurologist, psychoeducation, stress management and further specific treatment based on underlying psychological causes seems to be an effective way of treating this condition. Patients often require reassurance that their condition is real, and although psychological factors may be explored, their fits are not viewed as being imaginary. Any underlying psychiatric disorders, commonly affective or anxiety disorders, should be identified and treated as appropriate.

Any anti-epileptic medication should be tapered and discontinued. Appropriate use of various psychopharmacological agents is often necessary to treat underlying affective, anxiety or other psychiatric disorder.

\section{Neuropsychiatric aspects of epilepsy surgery}

Increasingly, refractory epilepsy is being treated successfully by surgical means. Patients with epilepsy who have not responded to two or more anti-epileptic drugs could be considered for epilepsy surgery. The rationale for surgery is the removal of the epileptogenic focus and the most common procedure is anterior temporal lobectomy. Other procedures include hippocampectomy and lesionectomy; multiple subpial transection is sometimes considered when a less damaging intervention is required in more sensitive areas of the brain. For patients with temporal lobe epilepsy or lesional epilepsy, seizure-free or near seizurefree rates as high as $80-90 \%$ have been achieved using surgery.

Post-epilepsy surgery neuropsychiatric problems mirror the overlap of psychiatric problems with epilepsy. Overall rates of neuropsychiatric problems following epilepsy surgery can be very 


\section{MCO answers}

$1 \mathrm{~b} \quad 2 \mathrm{e} \quad 3 \mathrm{~b} \quad 4 \mathrm{~d} \quad 5 \mathrm{~b}$ similar to rates in people with epilepsy in general. However, some patients can show improvement in pre-existing neuropsychiatric problems, whereas others can develop new problems. De novo psychosis is rarely seen post-epilepsy surgery (0.5-1\%). More commonly, about a third of patients can present with anxiety and depression 6-12 weeks after the surgery irrespective of surgery outcome. Foong $\&$ Flugel (2007) summarise the neuropsychiatric issues in relation to epilepsy surgery.

\section{Vagus nerve stimulation}

Patients who do not respond to anti-epileptic drugs and are either not eligible for or do not want epilepsy surgery could be considered for vagus nerve stimulation. It is considered as an adjunct treatment in partial seizures (localisation-related epilepsy). In the USA, vagus nerve stimulation has also been approved by the Food and Drug Administration for the treatment of major depression. (See Groves \& Brown (2005) for a review of application and mechanisms of action of vagus nerve stimulation.)

\section{Conclusions}

Both epileptic disorders and their treatment can affect the mental state of patients and produce a wide variety of symptoms. A range of mental health problems can be seen in patients with epilepsy that can lead on to significant distress, dysfunction and impair their quality of life. Neuropsychiatric problems in epilepsy are difficult to diagnose and they are frequently missed or overlooked. Successful treatment can have a profound effect on a patient's quality of life and may contribute towards better seizure control.

\section{References}

Alper RK, Barry JJ, Balabanov AJ (2002) Treatment of psychosis, aggression, and irritability in patients with epilepsy. Epilepsy and Behavior 3: S13-8.

American Psychiatric Association (1994) Diagnostic and Statistical Manual of Mental Disorders (4th edn) (DSM-IV). APA.

Barry J (2003) The recognition and management of mood disorders as a comorbidity of epilepsy. Epilepsia 44 (suppl 4): 30-40.

Benbadis SR, Allen HW (2000) An estimate of the prevalence of psychogenic non-epileptic seizures. Seizure 9: 280-1.

Beyenburg S, Mitchell AJ, Schmidt D, et al (2005) Anxiety in patients with epilepsy: Systematic review and suggestions for clinical management. Epilepsy and Behavior 7: 161-71.

Boylan LS, Flint LA, Labovitz DL, et al (2004) Depression but not seizure frequency predicts quality of life in treatment resistant epilepsy. Neurology 62: 258-61.

Blumer D (1992) Postictal depression: significance for the neurobehavioral disorder of epilepsy. Journal of Epilepsy 5: 214-9.

Blumer D, Montouris G, Davies K (2004) The interictal dysphoric disorder: recognition, pathogenesis, and treatment of the major psychiatric disorder of epilepsy. Epilepsy and Behavior 5: 826-40.
Christensen J, Vestergaard M, Mortensen PB, et al (2007) Epilepsy and risk of suicide: a population-based case - control study. Lancet Neurology 6: 693-8.

Devinsky 0, Vazquez B (1993) Behavioral changes associated with epilepsy. Neurologic Clinics 11: 127-49.

Devinsky 0 (2003) Psychiatric comorbidity in patients with epilepsy: implications for diagnosis and treatment. Epilepsy and Behavior 4: S2-10.

Edeh J, Toone B (1987) Relationship between interictal psychopathology and the type of epilepsy. Results of a survey in general practice. British Journal of Psychiatry 151: 95-101.

Ettinger AB, Reed ML, Goldberg JF, et al (2005) Prevalence of bipolar symptoms in epilepsy vs other chronic health disorders. Neurology 65 : 535-40.

Ficker DM (2000) Sudden unexplained death and injury in epilepsy. Epilepsia 41 (suppl 2): S7-12.

Foong J, Flugel D (2007) Psychiatric outcome of surgery for temporal lobe epilepsy and presurgical considerations. Epilepsy Research 75: 84-96.

Forsgren L (1992) Prevalence of epilepsy in adults in northern Sweden. Epilepsia 33: 450-8

Gaitatzis A, Carroll K, Majeed A, et al (2004) The epidemiology of the comorbidity of epilepsy in the general population. Epilepsia 45 : 1613-22.

Gates JR (2002) Nonepileptic seizures: classification, coexistence with epilepsy, diagnosis, therapeutic approaches and consensus. Epilepsy and Behavior 3: 28-33.

Gaynor D, Cock H, Agrawal N (2009) Psychological treatments for functional non-epileptic attacks: a systematic review. Acta Neuropsychiatrica 21: $158-68$

Goldensohn ES, Porter RJ, Schwartzkroin PA (1997) The American Epilepsy Society: an historic perspective on 50 years of advances in research. Epilepsia 38: 124-50.

Groves DA, Brown VJ (2005) Vagal nerve stimulation: a review of its applications and potential mechanisms that mediate its clinical effects. Neuroscience and Biobehavioral Reviews 29: 493-500.

Hauser WA, Annegers JF, Elveback LR (1980) Mortality in patients with epilepsy. Epilepsia 21: 399-412.

International League Against Epilepsy (1989) Commission on Classification and Terminology: Proposal for revised classification of epilepsies and epileptic syndromes. Epilepsia 30: 389-99.

Kanner AM (2003) Depression in epilepsy: prevalence, clinical semiology, pathogenic mechanisms, and treatment. Biological Psychiatry 54: 388-98

Kanner AM, Kozak AM, Frey M (2000) The use of sertraline in patients with epilepsy: is it safe? Epilepsy and Behavior 1: 100-5.

Krishnamoorthy ES, Trimble MR, Sander JWAS, et al (2002) Forced normalisation at the interface between neurology and Psychiatry. Epilepsy and Behavior 3: 303-8.

Krohn W (1961) A study of epilepsy in northern Norway: its frequency and character. Acta Psychiatrica Scandinavica Supplementum 36 : 215-25.

Kwan P, Brodie MJ (2000) Early identification of refractory epilepsy. New England Journal of Medicine 342: 314-9.

Lambert MV, Robertson MM (1999) Depression in epilepsy: etiology, phenomenology, and treatment. Epilepsia 40 (suppl 10): S21-47.

Landolt H (1953) Some clinical EEG correlations in epileptic psychoses (twilight states). Electroencephalography and Clinical Neurophysiology 5: 121.

Lopez-Rodriguez F, Altshuler L, Kay J, et al (1999) Personality disorders among medically refractory epileptic patients. Journal of Neuropsychiatry and Clinical Neurosciences 11: 464-9.

Mensah SA, Beavis JM, Thapar AK, et al (2007) A community study of the presence of anxiety disorder in people with epilepsy. Epilepsy and Behavior 11: 118-24. 
Mula M, Trimble MR (2003) Pharmacokinetic interactions between antiepileptic and antidepressant drugs. World Journal of Biological Psychiatry 4: 21-4.

Mula M, Jauch R, Cavana A, et al (2008) Clinical and psychopathological definition of the interictal dysphoric disorder of epilepsy. Epilepsia 49 : 650-6.

National Collaborating Centre for Mental Health (2004) The Epilepsies: Diagnosis and Management of the Epilepsies in Adults and Children in Primary and Secondary Care (Clinical Guideline CG020). National Institute for Clinical Excellence.

Nilsson L, Ahlbom A, Farahmand Y, et al (2002) Risk factors for suicide in epilepsy: a case control study. Epilepsia 43: 644-51.

Prueter C, Norra C (2005) Mood disorders and their treatment in patients with epilepsy. Journal of Neuropsychiatry and Clinical Neurosciences 17: 20-8.

Robertson MM, Trimble MR (1985) The treatment of depression in patients with epilepsy. A double-blind trial. Journal of Affective Disorders 9: $127-36$

Ropper AH, Brown RH (2005) Adams and Victor's Principles of Neurology (8th edn). McGraw-Hill.

Sachdev P (1998) Schizophrenia-like psychosis and epilepsy: the status of the association. American Journal of Psychiatry 155: 325-36.

Shen W, Bowman ES, Markand ON (1990) Presenting the diagnosis of pseudoseizure. Neurology 40: 756-9.
Szaflarski JP, Ficker DM, Cahill WT, et al (2000) Four year incidence of psychogenic nonepileptic seizures in adults in Hamilton County, $\mathrm{OH}$. Neurology 55: 1561-3.

Tellenbach H (1965) Epilepsie als Anfallsleiden und als Psychose: uber alternative Psychosen paranoider pragung bei "forcierter Normalisierung" (Landolt) des Elektroencephalogramms Epileptischer. Der Nervenarzt 36: 190-202.

Thaper A, Roland M, Harold G (2005) Do depression symptoms predict seizure frequency - or vice versa? Journal of Psychosomatic Research 59: 269-74.

Toone BK (2000) The psychoses of epilepsy. Journal of Neurology, Neurosurgery and Psychiatry 69: 1-3.

Torta R, Keller R (1999) Behavioral, psychotic and anxiety disorders in epilepsy: etiology, clinical features, and therapeutic implications. Epilepsia 40 (suppl 10): S2-20.

Viikinsalo M, Sawrie S, Kuzniecky RI, et al (2000) AES Proceedings. Epilepsia 41 (suppl 7): 175.

World Health Organization (1992) The ICD-10 Classification of Mental and Behavioural Disorders: Clinical Descriptions and Diagnostic Guidelines. WHO.

World Health Organization (2005) Atlas: Epilepsy Care in the World. WHO

Zaber JE, Copeland LA, Amuan A, et al (2007) The role of co-morbid psychiatric conditions in health status in epilepsy. Epilepsy and Behavior 10: 539-46.

\section{MCOs}

Select the single best option for each question stem

1 Which of the following is not a risk factor for psychopathology in epilepsy:

a young age at onset of epilepsy

b preictal EEG abnormalities

c neuropathological damage to basal ganglia

d low socioeconomic status

e overprotection by families.
3 As regards functional non-epileptic attacks:

a they are commonly associated with abnormal electrical brain discharges

b they may occur in people who have epilepsy

c injury during the fit suggests epilepsy rather than a functional non-epileptic attack

$\mathrm{d}$ increased prolactin $2 \mathrm{~h}$ after a fit is a diagnostic feature of such an attack

e home videos of attacks are of no use diagnostically.

4 In relation to depression in epilepsy:

a postictal depression is the most common presentation of depression in epilepsy

b preictal depression usually requires treatment with antidepressant medication

c frequency of seizures affects patients' quality of life more than depression does d untreated depression could lower the seizure threshold

e ECT is contraindicated.

5 In relation to interictal psychosis of epilepsy:

a patients frequently exhibit negative symptoms of schizophrenia

b premorbid personality is commonly unaffected

c forced normalisation is the main explanation behind this condition

d antipsychotic drugs raise the risk of increased seizure frequency and are contraindicated

e the risk of clozapine to provoke seizures does not appear to be dose-dependent. 